\title{
A HIGH SPEED PHOTOMETRIC SURVEY OF NORMAL AND PECULIAR A-TYPE STARS
}

\author{
O.M. KURTANIDZE AND M.G.NIKOLASHVILI \\ Astronomical Observatory, 383762 Abastumani, \\ Republic of GEORGIA \\ okur@abao.kheta.ge,maria@abao.kheta.ge
}

\begin{abstract}
The programme of long-term high-speed photometric survey about two hundred Normal and Peculiar A-type stars with $125 \mathrm{~cm}$ RC telescope equipped by Two-Star Photometer is presented.
\end{abstract}

\section{INTRODUCTION}

Rapidly oscillating Ap stars form a unique group of objects having pulsations with frequencies close to those seen in the Sun, but with amplitudes several times larger. They are chemically peculiar which pulsate in low-degree $(l<3)$, high-overtone $(n>>l)$ non-radial p-modes with periods in the range of $4-15 \mathrm{~min}$. The peak-to-peak amplitudes are $\leq 16 \mathrm{mmag}$. The searches for pulsations in Ap stars were summarized in the recent reviews (Kurtz, 1990; Martinez, 1993; Martinez and Kurtz, 1995).

\section{ABASTUMANI SURVEY}

Prior beginning of the Abastumani survey, only 27 roAp stars were discovered since 1978. Most of roAp stars discovered (25) are located in the southern hemisphere. Two roAp located in the northern hemisphere $10 \mathrm{Aql}$ and $\gamma$ Equ are very bright, brighter than 6mag which definitely indicates that all northern sky photometric surveys of roAp stars were very ineffective. In order to ameliorate this situation we have initiated a long-term high-speed photometric survey of the peculiar and normal A-F stars in the northern hemisphere. The scientific goal of the survey are as follows.

1. To increase the number of known roAp in the norhtern hemisphere, so that the extent of the roAp phenomenon can be better determined.

2. To apply the techniques of asteroseismology to these new roAp stars.

3. To study the relation between the roAp stars and other pulsating stars in the same region of the H-R diagram, such as $\delta$ Sct stars.

The candidates in our survey are drawn from the Catalogue General des Etoiles Ap et Am (Renson 1991) and the Michigan Spectral Survey. The instrument used was 
the Two-Star Photometer attached to the $125 \mathrm{~cm}$ RC telescope (1/13) of Abastumani Astrophysical Observatory. It permits to carry out simultaneous measurements of two objects in the field $22 \times 22$ sq.min with exposure of $10^{-3}$ to $10^{2}$. Photometer operates on line with $\mathrm{PC} / \mathrm{AT}$ computer.

In principle, the use of Two-Star photometers in high-speed photometry should make it possible to eliminate sky transparency and sky background variations to produce scintillation limited amplitude spectra at all frequences. But it is usually difficult to acquire a comparison star of similar brightness in the limited field of second channel. Hence, if the program star data are normalized to the comparison star data on a point-by-point basis, the large photon noise of the comparison star data degrades the quality of the program star data. We smoothed the comparison star data over half the period of interest in the program star and then normalized the program star data with the comparison star data, assuming that the photon noise for comparison star is less than sky transparency noise when integrated over half the period of interest in the program star (see Kurtz, 1984). On nights of significantly good quality the individual channels can be analysed separetely. This allows more thorough interpretation of the data since each star produces its own result that can be used along with the combined channels, making identification and interpretation of significant periodicities more straightforward.

The duration of a typical data set is $2.5-3 \mathrm{~h}$. Data set significantly shorter than these do not produce periodograms of sufficient quality or resolution for the periods of interest here. On the other hand, to produce an extremely high resolution periodogram, much more than $3 \mathrm{~h}$ of observations are required. If multiple frequenes are present, time series longer than any beat frequences are required to resolve all components. The 20 or 30 arcsec diameter apertures and integration times 2-10 sec were used. The observations are interrupted at regular intervals for measurements of the sky background and dark. The software for data processing includes the Fourier filtering and fine spectral scanning methods for periodic fast phenomena and the correlation analysis for non-periodic variations of light curves.

$\mathrm{Up}$ to now we have searched nine $\mathrm{SrCrEu}$ stars for high-overtone pulsations. Seven of them were observed on more than one night. Unfortunately none of these stars show variations, although we have precision required to reveal the presence of oscillations even for lower amplitude roAp stars. Several roAp stars may lie undiscovered in these null results because we happend to observe them near quadrature (amplitude minimum) or during a time when several oscillation frequences in the star interfered destructively.

Unfortunately at present due to very seldom electricity supply of the Observatory we are unable to carry out the Programme effectivelly.

\section{References}

Kurtz, D.W., 1984, NASA CP-2350, 56.

Kurtz, D.W., 1990, ARA\&A, v.28, 607.

Martinez, P., Kurtz, D.W., 1995, ASP CS v.83, 58.

Martinez, P., 1993a, Ph.D.Thesis, Univ. of Cape Town.

Renson, P., 1991, Catalogue General des Etoiles Ap et Am. 